\title{
Amorphous Salts Solid Dispersions of Celecoxib: Enhanced Biopharmaceutical Performance and Physical stability
}

Sumit Mukesh ${ }^{1}$, Prachi Joshi ${ }^{1}$, Arvind K. Bansal ${ }^{1}$, Mahesh Chand Kashyap ${ }^{1}$, Sanjay K. Mandal $^{2}$, Vasant Sathe ${ }^{3}$, and Abhay T. Sangamwar ${ }^{1}$.

1. Department of Pharmaceutics, National Institute of Pharmaceutical Education and Research, Sector-67, S.A.S. Nagar-160062, Punjab, India.

2. Department of Chemical Sciences, Indian Institute of Science Education and Research, Mohali, Sector-81, S.A.S. Nagar -140306, Punjab, India.

3. University Grant Commission-Department of Atomic Energy Consortium for Scientific Research, University Campus, Indore-452017, Madhya Pradesh, India.

\section{*Corresponding author}

Abhay T. Sangamwar

Tel: +91-0172 2214682;

Fax: +91-0172 2214692

E-mail: abhays@niper.ac.in,

abhaysangamwar@gmail.com 


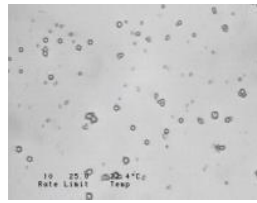

(a)

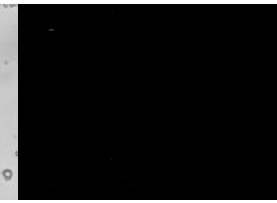

(b)

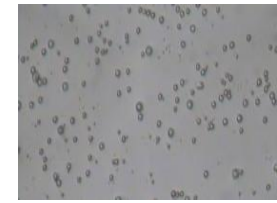

(c)

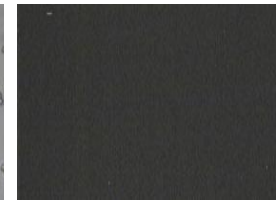

(d)

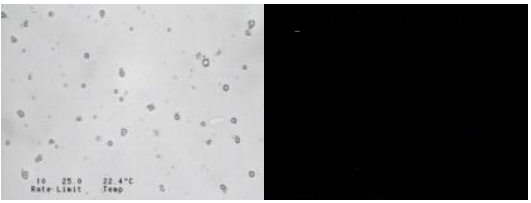

(e)

(f)

Figure S1. (a) Optical and (b) polarized light microscopy images of CEL-Na-Soluplus ASSD, (c) and (d) optical and polarized light microscopy of CEL-K-Soluplus ASSD, and (e) and (f) optical and polarized light microscopy images of binary CEL-Soluplus ASD.

\section{Data from long term stability studies.}

Microscopy images of samples after more than 11 months (352 days)

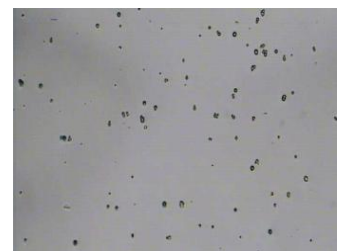

(a)

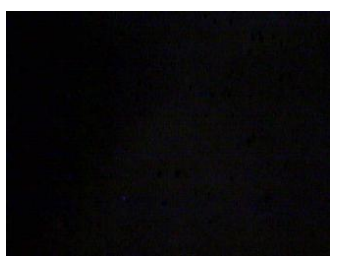

(b)

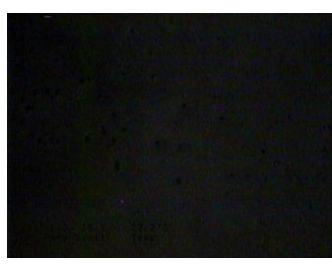

(c)

Optical (a) and polarized light microscopy (b) and (c) images of polymeric amorphous CEL-Na-Soluplus solid dispersion

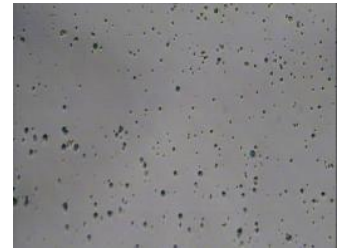

(d)

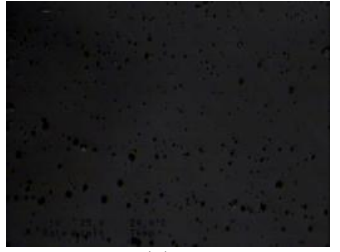

(e)

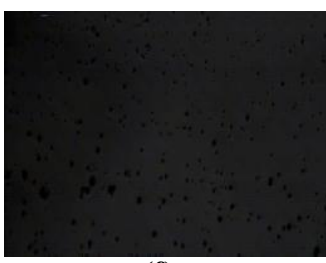

(f)

Optical (d) and polarized light microscopy (e) and (f) images of polymeric amorphous CEL-K-Soluplus solid dispersions

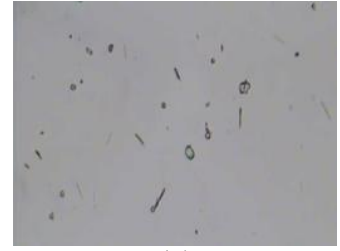

(g)

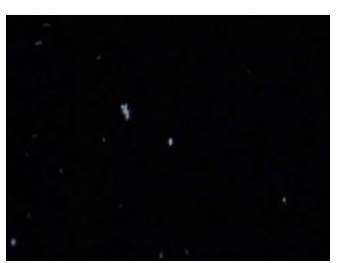

(h)

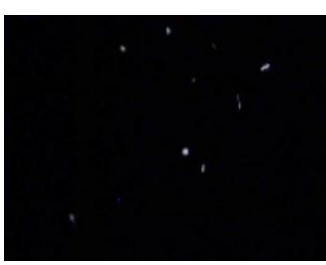

(i)

Optical (a) and polarized light microscopy (b) and (c) images of binary CEL-Soluplus amorphous solid dispersions

Figure S2. Optical and polarized light microscopy of polymeric amorphous CEL-salts solid dispersions and binary amorphous solid dispersion. 


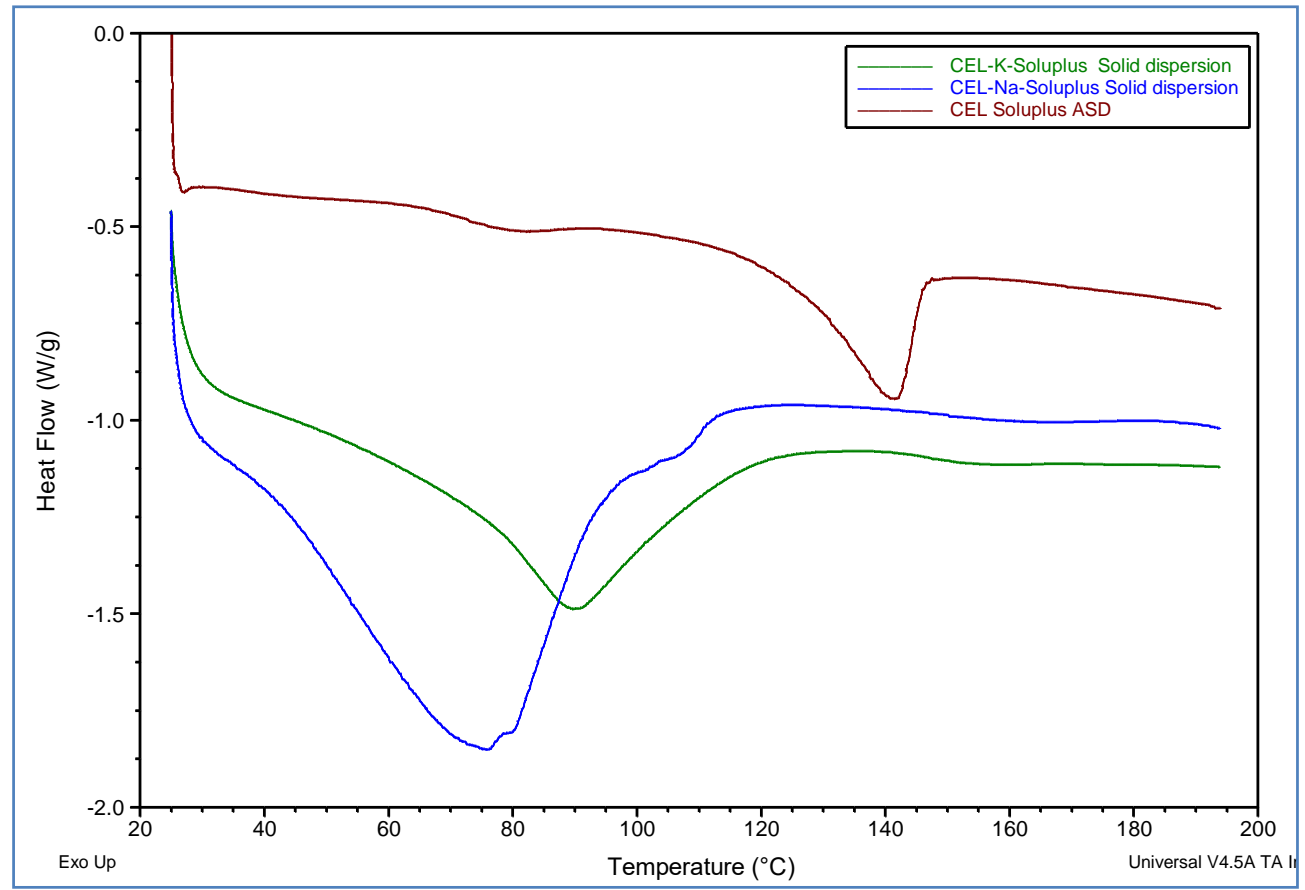

Figure S3. DSC analysis of 11 months old samples of polymeric amorphous CEL-Na-Soluplus and CEL-K-Soluplus solid dispersions, and binary CEL-Soluplus ASD.

Polymeric amorphous CEL-Na and CEL-K salt solid dispersions showing broad endotherms due presence of residual solvent and no melting endotherms were observed. Whereas, in case of CEL-Soluplus ASD, melting endotherm was observed indicating crystallization of ASD. 